\title{
Ondes et marnages dans les biefs dûs au remplissage et à la vidange des écluses
}

\author{
Lock-filling and emptying waves \\ and level variations in canal reaches
}

\author{
J. Michel
}

Compagnie Nationale du Rhône

\section{Introduction}

Le dimensionnement d'une voie navigable est fait de façon à assurer des conditions de navigation les meilleures possibles aux plus grands convois pris en compte dans le projet.

Toutefois, le confort de navigation peut être affecté par le remplissage et la vidange des écluses dont l'influence risque d'être d'autant plus forte que la section des canaux est réduite, et que la longueur des biefs n'est pas très grande. Par le jeu des réflexions et éventuellement de la mise en résonance des masses liquides, on peut aboutir à des conditions de navigation difficiles.

Il convient que l'évolution du plan d'eau reste dans des limites acceptables avec $0,30 \mathrm{~m}$ comme hauteur maximale d'une onde et $1 / 1.000^{\mathrm{e}}$ comme pente maximale associée du plan d'eau.

Ces phénomènes rapides masquent parfois un autre problème pouvant lui aussi conduire à des difficultés de navigation. En effet, la consommation en eau des écluses se traduit généralement par une évolution lente du niveau de chaque bief surtout lorsque l'alimentation de la voie d'eau est déficitaire. Il convient d'apprécier ce marnage pour éviter de réduire le pied de pilote en certains endroits des biefs (sur le seuil aval des écluses notamment), ou le tirant d'air sous les ouvrages d'art.

\section{Les ondes d'éclusage}

Les opérations de remplissage et de vidange sont en fait réalisées avec le souci d'obtenir un cycle de fonctionnement des écluses le plus court possible. En effet, la capacité de transport d'une voie navigable est directement liée au temps de franchissement des écluses.

Dans le cas des écluses du Rhône par exemple, le remplissage ou la vidange du sas sont effectués en 6 ou 7 minutes environ. Or, aussi bien en amont qu'en aval, on n'a jamais observé d'ondes gênantes pour la navigation du fait des éclusages. C'est qu'en effet les canaux où sont implantées ces écluses, sont dimensionnés pour le débit turbiné, et les sections transversales atteignent alors 1000 à $2000 \mathrm{~m}^{2}$.

Le problème est donc sensiblement différent du cas des voies conçues uniquement pour la navigation. La section transversale y est beaucoup plus faible. Ainsi pour le futur canal Saône-Rhin, en dehors des tronçons de rivière canalisée, la section est de l'ordre de $200 \mathrm{~m}^{2}$.

La résolution de ce problème a fait l'objet d'un cheminement particulier :

- une méthode expérimentale a été mise en cuvre, en réalisant des essais sur un tronçon de canal existant; - un modèle mathématique a été par la suite développé pour retrouver les résultats expérimentaux.

\section{Les mesures sur le canal du Nord}

C'est un des biefs du canal du Nord qui a été retenu pour l'expérimentation. Il est situé au Nord de Compiègne, entre les écluses n ${ }^{\circ} 16$ et 17 . Sa longueur est de $5630 \mathrm{~m}$. La figure 1 montre la configuration des lieux. Le canal présente sur un kilomètre de longueur environ une courbe assez prononcée de $500 \mathrm{~m}$ de rayon. Il est franchi par 4 ponts au droit desquels la section $\mathrm{du}$ canal est réduite. La section courante d'environ $80 \mathrm{~m}^{2}$ est trapézoïdale; elle présente normalement les caractéristiques suivantes:

$-22 \mathrm{~m}$ de largeur au plafond ;

$-3 / 2$ comme pente des talus;

$-3 \mathrm{~m}$ de mouillage normal.

Dix points de mesure des niveaux, enregistrés en continu, ont été installés sur ce bief, permettant de suivre la propagation et la déformation des ondes. 


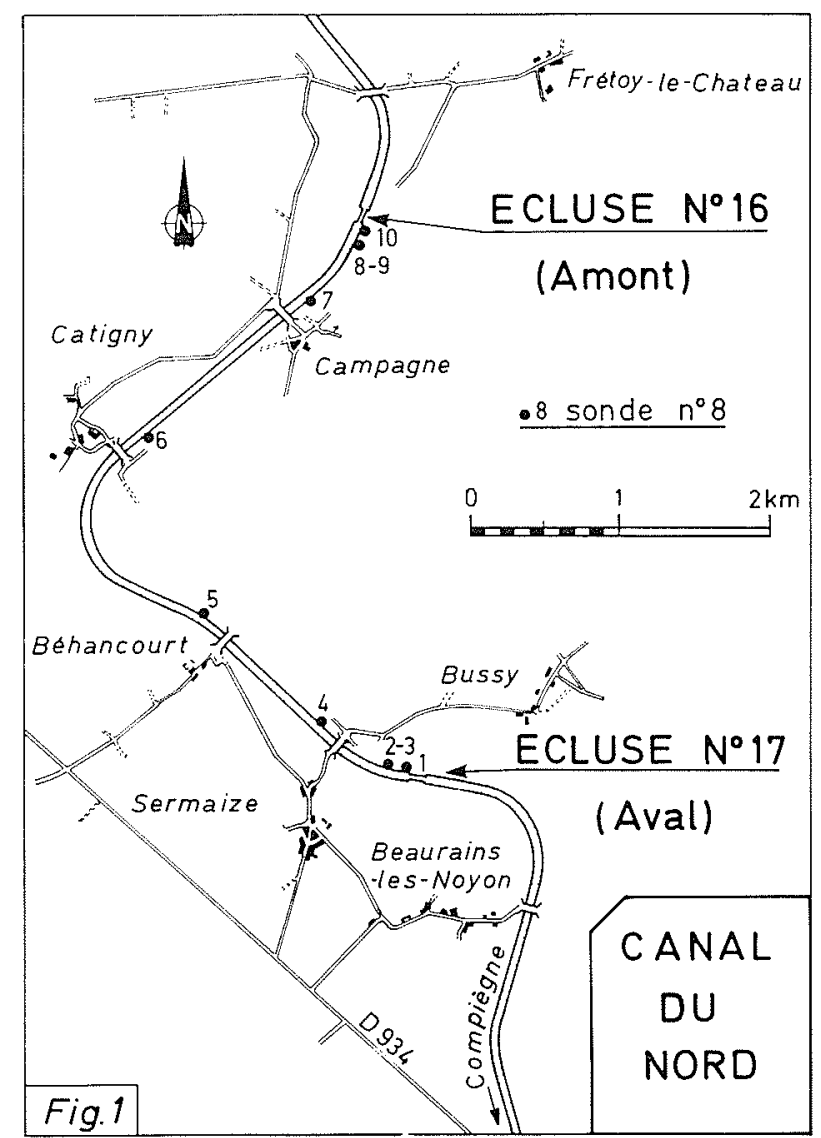

Les lois de vidange et de remplissage des écluses $\mathrm{n}^{\circ} 16$ et 17 ont été obtenues à l'aide de limnigraphes placés dans le sas.

Le but des essais était donc:

- de créer des ondes pour en étudier la forme et la transmission,

- de voir s'il était possible de mettre la masse liquide du bief en résonance.

Pour cela les essais se sont déroulés en effectuant la succession des mancuvres suivantes :

1) vidange de l'écluse $n^{\circ} 16$ amont;

2) vidange de l'écluse $n^{\circ} 16$ au moment du retour de l'onde après réflexion sur l'écluse $\mathrm{n}^{\circ} 17$;

3) remplissage de l'écluse $n^{\circ} 17$ aval, avant l'arrivée du deuxième train d'ondes.

Les lois de débit apparaissent sur la figure 2. On constate que le débit maximum injecté dans le bief lors de la vidange de l'écluse $\mathrm{n}^{\circ} 16$ atteint $55 \mathrm{~m}^{3} / \mathrm{s}$ environ, alors qu'il n'a pas été possible d'obtenir plus de $20 \mathrm{~m}^{3} / \mathrm{s}$ au remplissage de l'écluse $n^{\circ} 17$ par suite des sécurités existantes.

La figure 3 montre l'évolution des ondes en 6 points de mesures caractéristiques. On constate que l'onde initiale, de 0,30 $\mathrm{m}$ de hauteur s'amortit lentement. Elle se fractionne en une série d'ondulations successives au passage du coude, et, à partir de cet endroit, l'amortissement de l'onde devient irrégulier, parfois masqué par les réflexions secondaires. Par la suite, on observe une hauteur en crête des ondulations de $0,40 \mathrm{~m}$ contre l'écluse $\mathrm{n}^{\circ} 17$ aval lors de la $1^{\text {ère }}$ réflexion, une hauteur
MESURES D'ONDES DANS LE BiEF 16.17 DU CANAL DU NORD
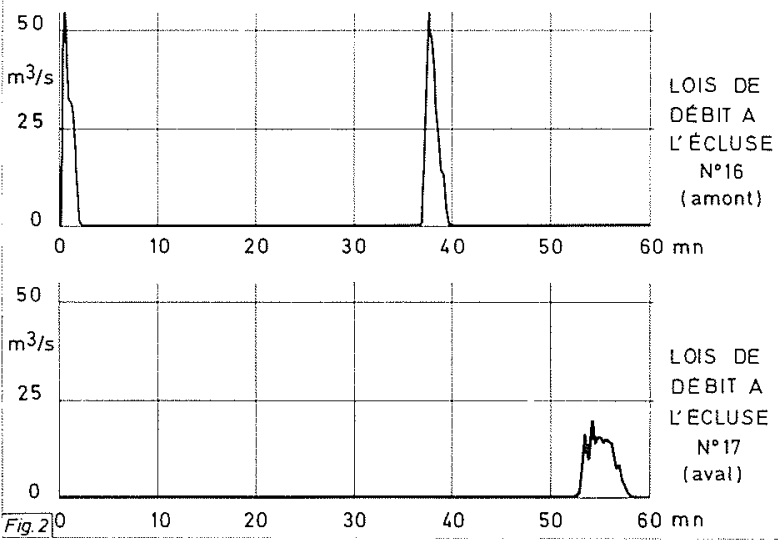

MESURES D'ONDES DANS LE BIEF $16-17$

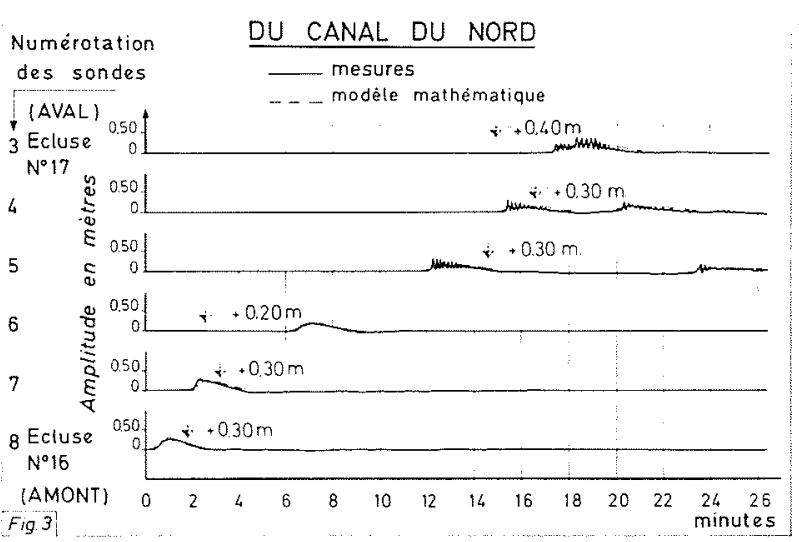

d'ondes de $0,40 \mathrm{~m}$ au moment de la $2^{\mathrm{e}}$ vidange de l'écluse $\mathrm{n}^{\circ} 16$ et une hauteur d'ondes de $0,30 \mathrm{~m}$ juste avant la $2^{\mathrm{e}}$ réflexion à l'écluse $\mathrm{n}^{\circ} 17$.

La mise en résonance de la masse liquide a bien été obtenue. Avant la $2^{\mathrm{e}}$ arrivée à l'écluse $\mathrm{n}^{\circ} 17$, on a observé un léger débordement sur le chemin de halage côté Sermaize ainsi qu'un déversement sur la porte amont de l'écluse.

Le risque d'onde de forte hauteur, avec mise en résonnance du plan d'eau est donc bien réel. Il est d'ailleurs parfois observé en fonctionnement normal d'une voie d'eau, où une opération sur une écluse peut venir se superposer à un état de surface pas très favorable. Des ruptures d'amarres se produisent de temps à autre à la suite de ce phénomène.

\section{Simulation sur modèle mathématique}

La méthode généralement utilisée pour le calcul des ondes rapides est fondée sur l'intégration numérique du système d'équations de Saint-Venant selon un schéma explicite. Il n'est pas question ici de présenter un tel programme de résolution, mais d'examiner plutôt ses résultats qui ont été reportés sur le même graphique en superposition avec les mesures in situ.

On constate que la corrélation est bonne, avec toutefois un certain lissage des ondes qui sont comparables à la moyenne des oscillations enregistrées. Le modèle 
mathématique a bien mis en évidence le faible amortissement des ondes et la mise en résonance de la masse liquide. Grâce à lui, il est donc possible de déterminer avec une bonne précision les mouvements de plan d'eau créés par les éclusages pour une voie navigable quelconque.

\section{La liaison Saône-Rhin}

La même méthode de calcul a donc été mise en œuvre lors des études de définition de la future voie navigable Saône-Rhin. Tous les biefs de cette liaison ont été examinés et l'étude a montré que ce sont ceux du versant Alsacien qui sont les plus sensibles à ce phénomène du fait de leur dimensionnement : les biefs sont en moyenne plus courts et la section transversale d'environ $200 \mathrm{~m}^{2}$, correspond uniquement à un chenal de navigation.

Dans ces conditions, le modèle mathématique a permis d'établir :

- que le débit maximum d'échange entre le sas et les biefs adjacents est d'environ $100 \mathrm{~m} 3 / \mathrm{s}$, pour limiter la hauteur des ondes à $0,30 \mathrm{~m}$;

- qu'il ne faut pas avoir un gradient de débit supérieur à $1,6 \mathrm{~m}^{3} / \mathrm{s} / \mathrm{s}$, pour limiter la pente du plan d'eau à $1 / 1000^{\mathrm{e}}$.

Ces deux résultats ont été utilisés lors de l'étude théorique du remplissage et de la vidange du sas des écluses de la liaison Saône-Rhin.

Quatre modes de fonctionnement différents ont été testés pendant une heure de temps réel, lors de l'étude des ondes:

$1^{\text {er }}$ : vidange de l'écluse amont;

$2^{\mathrm{e}}$ : remplissage de l'écluse aval ;

$3^{\mathrm{e}}$ : vidange et remplissage simultanés;

$4^{\mathrm{e}}$ : vidange et remplissage décalés d'une onde.

Le coefficient de Strickler du canal, adopté pour le calcul, est de 60 . Les résultats obtenus appellent les observations suivantes:

1) l'onde initiale est toujours inférieure à $25 \mathrm{~cm}$; 2) quel que soit le mode de fonctionnement, l'onde maximale est toujours produite par la première réflexion;

3) l'amortissement des ondes est très long, sauf dans le cas de la vidange et du remplissage décalés d'une onde. Les mouvements de plan d'eau sont encore importants après une heure d'observation dans les autres cas; 4) le dimensionnement du canal a montré que la forme de section la plus avantageuse vis à vis des efforts de poussage est celle qui a les talus les plus raides possible. Deux types de sections de superficies équivalentes ont été retenus avec des talus en $2 / 1$ et en $3 / 1$, celle avec les talus en $2 / 1$ ayant un rayon hydraulique un peu plus grand. Il s'en suit que le coefficient de pertes de charge $J=V^{2} / K^{2} R^{4 / 3}$ est plus faible pour cette section en $2 / 1$. L'amortissement des ondes y est donc plus long que dans la section en $3 / 1$.

Ainsi, dans le cas de la vidange de l'écluse amont, l'onde maximale observée atteint $44 \mathrm{~cm}$ dans le bief de Balschwiller, tandis qu'une heure après, les surélévations du plan d'eau sont comprises entre 20 et $35 \mathrm{~cm}$.

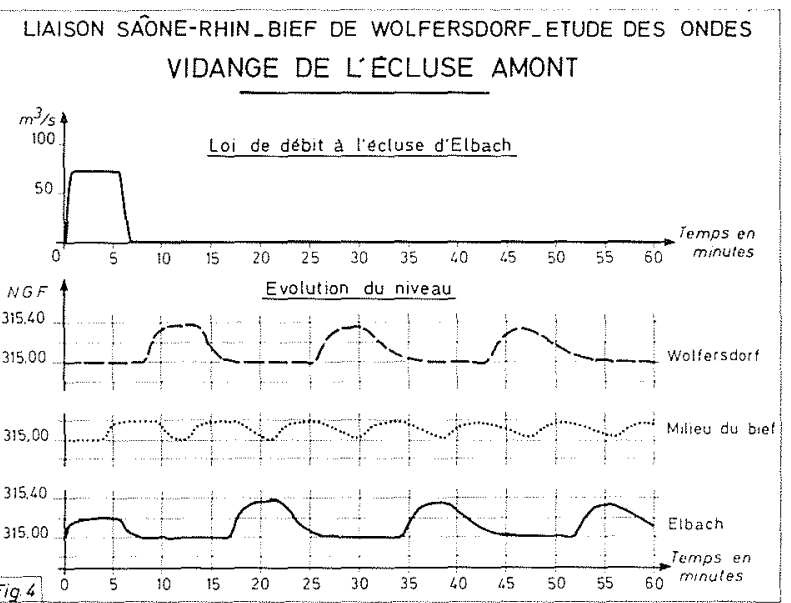

La figure $n^{\circ} 4$ présente la vidange de l'écluse d'Elbach dans le bief de Wolfersdorf. L'onde initiale, de $0,20 \mathrm{~m}$ de hauteur, passe à $0,38 \mathrm{~m}$ lors de la première réflexion et atteint encore $0,34 \mathrm{~m}$ au moment de la $6^{\mathrm{e}}$ réflexion, 55 minutes après le début de la vidange. L'amortissement est donc très faible et montre qu'il convient d'éviter de superposer une deuxième vidange de l'écluse d'Elbach. On voit cependant que la longueur d'un créneau défavorable n'excède pas 7 à 8 minutes.

On peut faire les mêmes observations que précédemment lors du remplissage de l'écluse aval.

La vidange et le remplissage simultanés provoquent dans tous les biefs du versant Rhin un véritable balancement du plan d'eau. Ce balancement reste encore important au bout d'une heure. La figure 5 illustre le cas du bief de Wolfersdorf où l'on peut constater que l'évolution du plan d'eau est encore de $60 \mathrm{~cm}$ une heure après. Cette situation peut rendre délicate l'accès des convois aux écluses. On remarque cependant que la durée d'un créneau défavorable n'excède pas, comme précédemment, 7 à 8 minutes, aussi bien pour l'écluse amont que pour l'écluse aval.

$\mathrm{Au}$ contraire, la vidange et le remplissage décalés d'une onde, permettent d'atténuer très rapidement les ondes comme on peut le voir sur la figure 6 (bief de Wolfersdorf). L'onde maximale (1ère réflexion) voit sa hauteur diminuer d'environ $30 \%$; au bout d'une

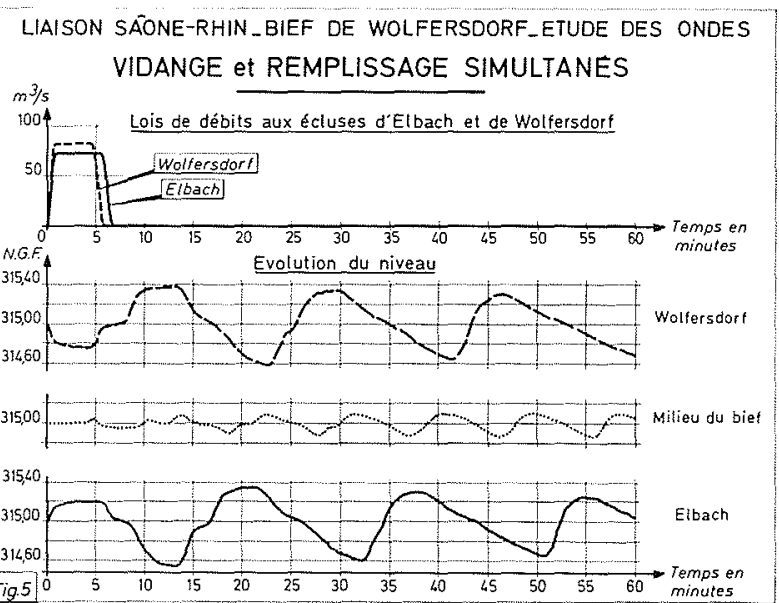




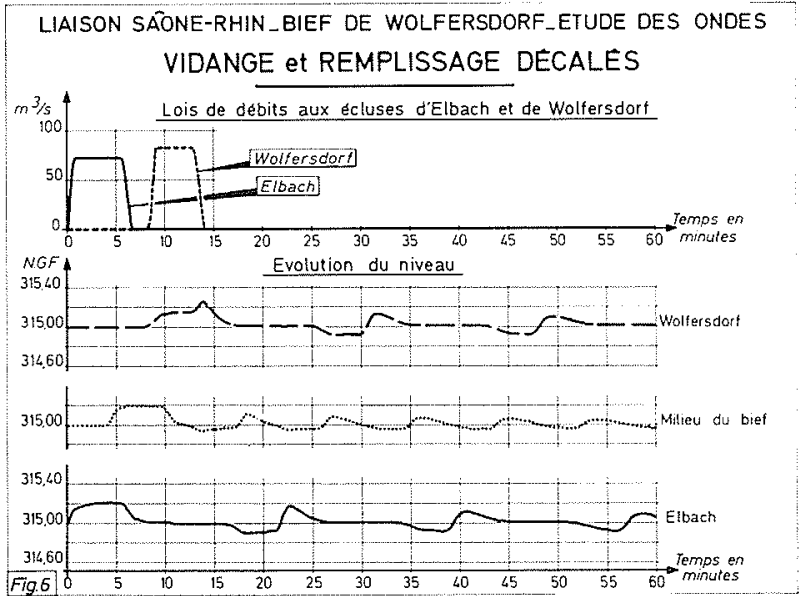

heure, l'évolution du plan d'eau est inférieure à $20 \mathrm{~cm}$ et cela est valable pour tous les biefs. Ce mode de fonctionnement est donc particulièrement intéressant. Il pourrait cependant conduire à un allongement appréciable du temps de transit des convois sur un tronçon de voie navigable. En réalité le problème devrait être moins important qu'il ne semble, au vu de ces résultats théoriques. En effet, les hauteurs d'ondes maximales apparaissent au droit des écluses dont la chute réduite est la plus forte. Or ces écluses sont accolées aux biefs les plus courts et le temps de transmission des ondes y est donc plus faible. L'attente d'un créneau favorable ne devrait donc pas être trop longue. De toutes façons, la durée d'un créneau défavorable ne dépasse pas 7 à $8 \mathrm{mi}$ nutes.

$\mathrm{Au}$ contraire, les biefs de grande longueur sont commandés, en général, par des écluses de chute plus faible, et les problèmes d'ondes sont moins importants. En tout état de cause, la durée d'un créneau défavorable est la même que précédemment.

Une étude systématique du fonctionnement des écluses d'une voie navigable, devrait ainsi permettre d'apprécier la longueur réelle des créneaux défavorables et de définir les pertes de temps maximales. Il est très raisonnable de penser que ces pertes de temps seront faibles.

Les calculs ont été aussi effectués en augmentant sensiblement la rugosité du canal, par le choix d'un Strickler de 40 . Les résultats restent très voisins et au bout d'une heure, l'écart sur les hauteurs d'onde est de l'ordre de $5 \mathrm{~cm}$. Il semble difficile de pouvoir obtenir pratiquement une rugosité plus forte, qui serait de toutes façons défavorables au déplacement des bateaux.

\section{Le marnage dans les biefs}

Le marnage correspond à un évolution lente et progressive du niveau moyen dans un bief.

Lorsqu'un bief est constitué d'un tronçon de rivière canalisé, ce marnage est essentiellement dù à l'hydrau licité et est pratiquement insensible à la consommation d'eau liée au trafic. Dans le cas d'une écluse noderne de $10 \mathrm{~m}$ de chute, de $195 \times 12 \mathrm{~m}$ de dimensions, lo volume d'eau perdu à chaque éclusée est de $23600 \mathrm{~m}^{3}$,

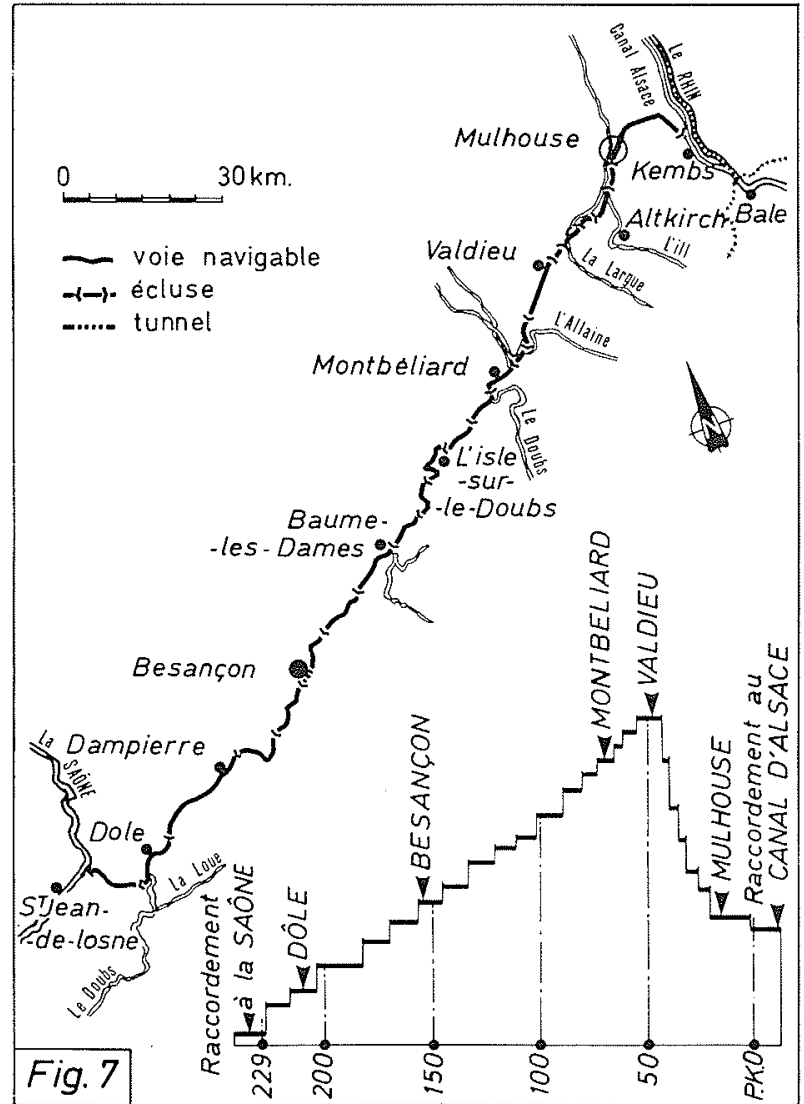

ce qui correspond à une consommation de $6,6 \mathrm{~m}^{3} / \mathrm{s}$ lors de la saturation de la voie, avec une durée du cycle d'une heure. Ce débit est faible et son influence sera d'autant plus réduite s'il existe une écluse à chaque extrémité du bief, le débit consommé par l'écluse aval pouvant être atténué dans d'assez grandes proportions par le débit injecté par l'écluse amont. Cela n'est cependant pas vrai pour le bief de partage d'une voie navigable.

L'influence de la consommation en eau des écluses sera donc sensible lorsque la voie est uniquement dimensionnée pour la navigation et surtout si l'alimentation en eau du bief de partage est déficitaire.

Tel est le cas du versant Alsacien de la future liaison Saône-Rhin dont la figure 7 montre la vue en plan et le profil en long.

L'étude du marnage des biefs a été effectuée sur modèle mathématique.

\section{Les hypothèses du calcul}

Pour cela, il est nécessaire de normaliser les divers paramètres qui influent directement sur le marnage de la voie.

1) Le cycle de fonctionnement des écluses est décom. posé selon le schéma classique, suite aux études engagées à ce sujet. La figure 8 présente la décomposition du cycle de 60 minutes.

2) La durée journalière de fonctionnement de la voie peut varier de 0 à $24 \mathrm{~h}$. Deux valeurs ont été examinées : 12 heures et 20 heures. 


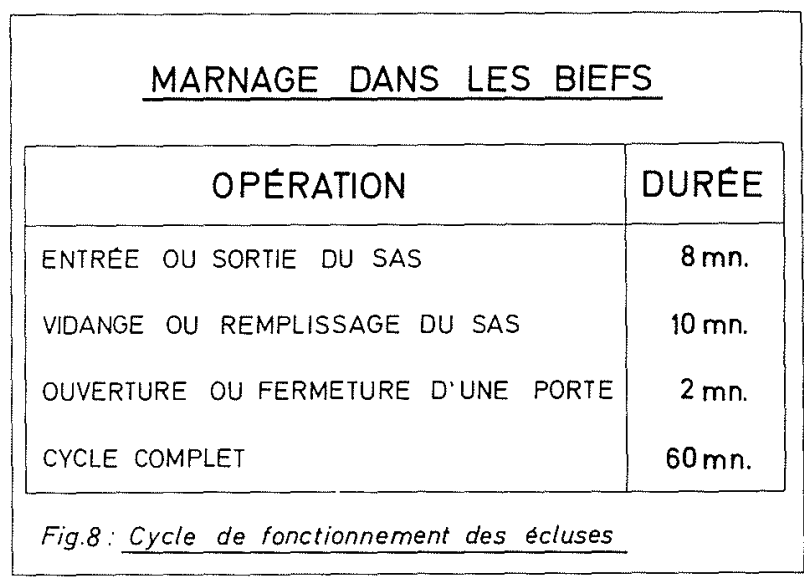

3) Le trafic constitue le paramètre le plus aléatoire de cette étude et un certain nombre d'hypothèses simplificatrices ont été faites qui concernent :

- la vitesse de déplacement des convois, choisie uniformément égale à $8,5 \mathrm{~km} /$ heure ;

- le déplacement des convois qui est observé dans un seul sens de marche. Cela suppose que les convois naviguant en sens inverse n'ont aucune influence sur le mouvement des bateaux à l'étude, notamment sur les temps d'attente aux écluses. Cela signifie que l'on a cherché à rendre maximum le nombre de bateaux franchissant la voie dans le sens privilégié et à obtenir ainsi une cadence de fonctionnement journalière des écluses la plus grande possible

4) La consommation des écluses est compensée par des pompages bief par bief, la voie étant supposée déficitaire en eau. Ces pompages sont réalisés en priorité aux 8 heures creuses. Ils peuvent être étendus aux heures pleines dans la limite d'une durée maximale de 20 heures.

5) La consommation en eau de chaque écluse tient compte de la présence éventuelle de bassins d'épargne. 6) La cote initiale du plan d'eau de chaque bief a été optimisée de façon à ce que le niveau le plus bas observé durant le fonctionnement soit supérieur de moins de $15 \mathrm{~cm}$ au niveau des plus basses eaux navigables.

\section{La liaison Saône-Rhin}

Le tronçon de canal étudié part du raccordement au canal d'Alsace jusqu'à l'aval de l'écluse de Froidefontaine, sur le bief de partage côté Doubs.

Le modèle détermine les temps d'attente de chaque convoi aux diverses écluses du tronçon. Il est ainsi rapidement apparu qu'il y a intérêt à choisir des durées de cycle identiques pour toutes les écluses, même pour celles où, du fait de la faible dénivellation, on aurait pu adopter des temps plus courts. Cela justifie le choix fait dans cette étude d'un cycle unique de 60 minutes.

Le cycle des écluses étant adopté, la longueur du tronçon à l'étude et la vitesse des bateaux déterminent, pour une durée de fonctionnement donnée, la distribution du trafic.

La figure 9 présente les marnages obtenus dans chaque bief pour 20 heures de fonctionnement de la voie

\begin{tabular}{|l|c|c|c|c|}
\hline \multicolumn{6}{|c|}{ LIAISON SAÔNE RHIN } \\
\hline Ecluses & $\begin{array}{c}\text { Chute } \\
\text { en m }\end{array}$ & $\begin{array}{c}\text { coef od } \\
\text { èpargne }\end{array}$ & $\begin{array}{c}\text { Chute } \\
\text { réduite } \\
\text { en } \mathrm{m} .\end{array}$ & $\begin{array}{c}\text { Marnage } \\
\text { en cm. }\end{array}$ \\
\hline NIFFER & 5.70 & 0 & 5.70 & 5 \\
BRUNSTATT & 14.00 & 0,357 & 9.00 & 5 \\
ZILLISHEIM & 9.00 & 0 & 9,00 & 10 \\
St BERNARD & 16.00 & 0,333 & 10.65 & 5 \\
BUETHWILLER & 16.00 & 0,333 & 10,65 & 7 \\
WOLFERSDORF & 24.00 & 0,555 & 10.65 & 5 \\
ELBACH & 21.80 & 0,511 & 10.65 & 13 \\
\hline Fig.9 : Marnages dans les biefs du versant Rhin \\
20h. de trafic et 20h. de pompage \\
\hline
\end{tabular}

et 20 heures de pompage. On note que les marnages observés sont réduits et très nettement inférieurs au marnage maximum admissible, incidences des ondes comprises, qui varie de $0,50 \mathrm{~m}$ à $0,90 \mathrm{~m}$ sur ce tronçon sauf pour le bief de Niffer où il est de $0,40 \mathrm{~m}$. Ce résultat découle du choix fait dans les coefficients d'épargne des écluses qui permet d'égaliser des chutes réduites. Les variations les plus fortes sont obtenues :

- dans le bief de partage avec 0,13 $\mathrm{m}$ du fait de l'absence de-débit d'apport gravitaire ;

- dans le bief de Zillisheim avec 0,10 $\mathrm{m}$ du fait de la différence de chute des écluses adjacentes;

- le cas du bief de Niffer est très satisfaisant malgré la disparité des chutes. Cela est dû à la grande longueur de ce bief (près de $23 \mathrm{~km}$ ).

Ainsi donc, le fonctionnement de cette voie navigable déficitaire en eau est tout à fait acceptable et permet de garantir pour chacun des biefs un mouillage et un tirant d'air corrects.

\section{Conclusion}

En définitive, le marnage dans les biefs dépend d'une façon déterministe d'un certain nombre de paramètres liés aux ouvrages et, d'une façon aléatoire, du trafic. Il est donc possible de l'évaluer seulement pour des situations particulières de la voie d'eau, à moins de disposer de prévisions convenables suffisamment à l'avance Ainsi les résultats du modèle mathématique précédent pourraient être infirmés dans le cas où, notamment, l'existance d'une zone portuaire sur un des biefs, conduirait à un trafic privilégié dans une direction, provoquant ainsi un déséquilibre dans le fonctionnement des écluses adjacentes; le marnage pourrait alors être plus élevé que celui obtenu selon la méthode précédente.

Cela montre qu'il convient d'adapter le modèle à l'ensemble des paramètres qui définissent la voie navigable. Leur nombre étant élevé, on voit l'intérêt qu'il y a à développer un automate chargé d'assurer la gestion de la voie avec pour but d'obtenir des marnages convenables et d'aboutir à une consommation en énergie électrique la plus faible et la moins chère possible.

Il serait alors intéressant de compléter cet automate par un système de détection des mouvements rapides des 
plans d'eau de façon à favoriser l'écrêtement des ondes tout en minimisant les pertes de temps aux écluses. Pour cela, il est nécessaire :

- d'interdire les manouvres conduisant à la conjonction de deux ondes de même signe, mais au contraire de les espacer dans le temps;

- de provoquer au contraire si possible la superposition d'ondes de signes opposés aux extrémités de chaque bief. L'écrêtement et l'amortissement des ondes est alors plus rapide.

Cette assistance dans le fonctionnement de la voie d'eau est importante et permettrait d'éviter le phénomène de mise en résonance des masses liquides dont dépend le confort et la sécurité de la navigation. 